\title{
Molecular characterization of Mycobacterium tuberculosis and Mycobacterium bovis isolates by Enterobacterial Repetitive Intergenic Consensus-PCR
}

\author{
Katiany Rizzieri Caleffi-Ferracioli ${ }^{1,2, *}$, Vanessa Pietrowski Baldin², Érika Noda Noguti², Vera Lucia \\ Dias Siqueira ${ }^{1}$, Regiane Bertin Lima Scodro ${ }^{1}$, Fernando Rogério Pavan ${ }^{3}$, Mario Hiroyuki Hirata ${ }^{4}$, \\ Rosilene Fressatti Cardoso ${ }^{1,2}$
}

\begin{abstract}
${ }^{1}$ Laboratory of Medical Bacteriology, Department of Clinical Analysis and Biomedicine, State University of Maringá, Paraná, Brazil, ${ }^{2}$ Postgraduation in Bioscience and Fisiopathology, State University of Maringá, Paraná, Brazil, ${ }^{3}$ School of Pharmaceutical Sciences, Department of Biological Sciences, Paulista State University, Araraquara, São Paulo, Brazil, ${ }^{4}$ School of Pharmaceutical Sciences, University of Sao Paulo, São Paulo, Brazil
\end{abstract}

\begin{abstract}
Tuberculosis (TB) is an infectious disease in which the molecular typing methods allow to have important information about the dynamics of transmission and to assist properly in disease control. Although the ERIC-PCR (Enterobacterial repetitive intergenic consensus-PCR) assay is fast and easy to perform, scarce studies have reported its use in epidemiological studies in TB outbreaks. In this study, we aimed to genotype Mycobacterium tuberculosis and $M$. bovis isolates by ERIC-PCR and compare its discriminatory power with two other classically used methods: 12 loci-MIRU (Mycobacterial Interspersed Repetitive Units) and Spoligotyping. The M. tuberculosis isolates studied were from northwestern and southwestern and M. bovis from northwestern Parana, Brazil. ERIC-PCR rendered banding patterns with great diversity ( 1 to 12 bands) of molecular sizes, ranging from 100 to $1600 \mathrm{bp}$. ERIC-PCR showed to be fast, simple and affordable to differentiate isolates. ERIC-PCR would be an important tool in the epidemiology of TB as screening in case of outbreak, which demands rapid intervention. However if any doubt persist, as it may occur with the application of only one genotypic method, other genotyping methods should be applied and carefully interpreted, always with additional epidemiological information.
\end{abstract}

Keywords: Tuberculosis. Molecular characterization. ERIC-PCR. Epidemiology.

\section{INTRODUCTION}

Tuberculosis (TB) is an infectious disease caused mainly by Mycobacterium tuberculosis and it has been known for centuries. In Brazil, it is estimated an incidence of $84 / 100.000$ in the population, in 2016, with a total of 81,137 cases notified (WHO, 2017).

Molecular differentiation of isolates is useful in epidemiological investigations as it is a means to better understand the mechanisms that influence the dynamics of transmission, identification of risk factors in a specific community and assist properly in disease control. The

\footnotetext{
*Correspondence: K. R. Caleffi-Ferracioli. Departamento de Análises Clínicas e Biomedicina, Universidade Estadual de Maringá. Avenida Colombo, 5790, 87020-900, Maringá, Paraná, Brazil. Tel. and Fax number: + 554430115375. E-mail: katianyrcf@gmail.com (K.R.Caleffi-Ferracioli)
}

genetic differentiation of $M$. tuberculosis complex isolates can determine the source of outbreaks and the relation between TB in domestic and wild animals, and identify the source of human infections (Mears et al., 2015).

A highly discriminatory typing method based on variations of the IS6110 specific sequences in the M. tuberculosis genome, the Restriction Fragment Lenght Polymorfism (RFLP) has been widely used in epidemiological studies of TB. However, despite the promising results, the RFLP is laborious, requiring highly skilled personnel to carry out the trial.

Spoligotyping, which is based on the amplification of a region containing multiple direct repeats (DR) in a highly polymorphic locus in the genome of bacillus belonging to the $M$. tuberculosis complex, brought valuable information on the evolution of the bacillus. At present, many studies have used the Mycobacterial 
Interspersed Repetitive Units (MIRU), such as the 12 lociMIRU, 15 loci-MIRU and 24 loci-MIRU, a PCR technique based on the study of Variable Number of Tandem Repeats sequences (VNTRs) that are repeated hundreds of times in the mycobacterial genome (Supply et al., 2000; Supply et al., 2001; Pandolfi et al., 2007; Machado et al., 2014).

The Enterobacterial Repetitive Intergenic Consensus sequence (ERIC) are repetitive elements of $126 \mathrm{bp}$ and appear to be restricted to transcribed regions of the bacterial chromosome, either in intergenic regions of polycistronic operons or in untranslated regions upstream or downstream of open reading frames. ERIC sequence was initially well-characterized in Gram negative bacillus belonging to the family Enterobacteriaceae and has been used to characterize clonal difference in species from this family. The presence of these sequences in mycobacteria was previously detected by some authors (Versalovic, Koeuth, Lupski, 1991; Englund, 2003; Sampaio et al., 2006a; Sampaio et al., 2006b), including in M. tuberculosis (Sechi et al., 1998). According to Sechi et al. (1998) and Sampaio et al. (2006a; 2006b), ERIC-PCR has sensitivity and is easy to be applied in epidemiological studies of TB outbreaks, especially when the IS6110 RFLP cannot help the differentiation or when the study involves other mycobacterial species.

Although ERIC-PCR is fast and easy to perform, scarce studies have reported its use in epidemiological studies in TB outbreaks. In this study, we aimed to characterize molecularly M. tuberculosis isolates by ERIC-PCR and compare its sensitivity with two other very used classical methods, MIRU and Spoligotyping.

\section{MATERIAL AND METHODS}

\section{Mycobacterium tuberculosis Complex isolates}

The M. tuberculosis clinical isolates used in this study were from patients attended at two independent laboratories at northwestern and southwestern Parana, Brazil. Isolates were previously biochemically identified (Kent, Kubica, 1985) and genotyped by MIRU and Spoligotyping (Machado et al., 2014; Noguti et al., 2010). Of the 93 isolates from northwestern Paraná, Brazil, which were previously typed by Spoligotyping and/or 12 loci-MIRU in our laboratory (Noguti et al., 2010), 73 were typed by Spoligotyping plus ERICPCR and 63 by 12 loci-MIRU plus ERIC-PCR (Table I). Spoligotyping of 73 isolates from the northwestern rendered 29 patterns, where 16 were unique and 13 clusters (57 isolates) with 2-10 isolates each one. The 12 loci-MIRU of 63 isolates rendered 52 patterns, where 47 were unique and 5 clusters ( 15 isolates) with 2-6 isolates each one. Spoligotyping of 83 isolates from the southwestern study (Machado et al., 2014) rendered 27 profiles, in which 16 were unique and 11 clusters (67 isolates) with 2-13 isolates each one. The 12 loci-MIRU

TABLE I - Discriminatory power of Enterobacterial Repetitive Intergenic Consensus sequence (ERIC-PCR), Spoligotyping and 12 loci-MIRU-VNTR typing, alone and in association in Mycobacterium tuberculosis

\begin{tabular}{lccccc}
\hline Methods / $\mathbf{n}^{\circ}$ isolates & $\mathbf{n}^{\circ}$ patterns & $\mathbf{n}^{\circ}$ unique & $\mathbf{n}^{\circ}$ clusters & $\begin{array}{c}\mathbf{n}^{\circ} \text { clustered } \\
\text { isolates }\end{array}$ & HGI $^{\mathbf{a}}$ \\
\hline Spoligotyping / 73 & & \multicolumn{5}{c}{ Northwestern } \\
12 loci-MIRU / 63 & 29 & 16 & 13 & 57 & 0.953 \\
ERIC-PCR / 73 & 52 & 47 & 5 & 15 & 0.989 \\
Spoligotyping + MIRU / 63 & 59 & 48 & 11 & 24 & 0.994 \\
ERIC-PCR + Spoligotyping / 73 & 57 & 53 & 4 & 10 & 0.996 \\
ERIC-PCR + 12 loci-MIRU / 63 & 72 & 71 & 1 & 2 & 0.999 \\
\hline & 63 & 63 & 0 & 0 & 1.0 \\
\hline Spoligotyping / 83 & & & Southwestern & & 0.993 \\
12 loci-MIRU / 83 & 27 & 16 & 11 & 67 & 0.997 \\
ERIC-PCR / 83 & 75 & 69 & 6 & 14 & 0.969 \\
Spoligotyping + MIRU / 83 & 41 & 25 & 16 & 58 & 0.999 \\
ERIC-PCR + Spoligotyping / 83 & 81 & 79 & 2 & 4 & 0.990 \\
ERIC-PCR + 12 loci-MIRU / 83 & 68 & 63 & 5 & 20 & 0.999 \\
\hline
\end{tabular}

a: HGI: Hunter-Gaston Index. 
of 83 isolates rendered 75 patterns, in which 69 were unique and 6 clusters (14 isolates) with 2-3 isolates each one.

The retrospective demographic and epidemiological data were collected for all patients in previous studies (Kent, Kubica, 1985; Noguti et al., 2010), by review of a national TB notification database (SINAN - National Diseases Notification System), being asked information such as: city and zip code of residence at the time of diagnosis, age, sex, ethnicity, HIV status, sample susceptibility profile, alcoholism and the occurrence of other diseases. The previous studies were approved by the Ethics Committee of the State University of Maringa, Parana (protocol $n^{\circ} 018 / 2011$, CAAE $n^{\circ}$ 0375.0.093.000-10).

The $17 \mathrm{M}$. bovis isolates, another species from the M. tuberculosis complex, were obtained from lymph node samples collected from bovines with macroscopic tuberculous-like lesions or positive skin test. Animals were selected during postmortem inspection in an abattoir in the northwestern Paraná, Brazil. The isolates were previously identified biochemically (Kent, Kubica, 1985) and genotyped by Spoligotyping (Figure 1). The $17 \mathrm{M}$. bovis isolates rendered 5 spoligopatterns, in which one was unique and 4 clusters (16 isolates) with 2, 2, 3 and 9 isolates.

\section{DNA extraction}

M. tuberculosis and M. bovis DNA were extracted from subcultures on Difco ${ }^{\mathrm{TM}}$ Lowenstein and Stonebrink media, respectively. Briefly, a loopful of bacterial growth was suspended in $6 \mathrm{M}$ guanidine hydrochloride (Sigma Chemical Co., St. Louis, Mo, USA) and bacilli were lysed by freezing at $-20{ }^{\circ} \mathrm{C}$ for $30 \mathrm{~min}$, followed by heating at $100{ }^{\circ} \mathrm{C}$ for $10 \mathrm{~min}$. This procedure was repeated twice. DNA was further extracted by 2 volumes of phenol- chloroform-isoamyl alcohol $(25: 24: 1, \mathrm{v} / \mathrm{v})$, followed by two steps of extractions with chloroform-isoamyl alcohol $(24: 1, v / v)$. DNA was purified by ethanol precipitation, dissolved in $50 \mu \mathrm{L}$ of Tris-EDTA, pH 8.0 (TE buffer) and stored at $-20{ }^{\circ} \mathrm{C}$ until use (González-Y-Merchand et al., 1996). DNA concentration was determined by ultraviolet spectrophotometry.

\section{ERIC-PCR}

ERIC-PCR was performed based on the analysis of Enterobacterial Repetitive Intergenic Consensus sequence according to Sechi et al. (1998) in M. tuberculosis clinical isolates from northwestern (Noguti et al., 2010) and southwestern (Machado et al., 2014), and in $M$. bovis isolates from northwestern, Paraná, Brazil. The amplification of the sequence in $M$. tuberculosis and $M$. bovis isolates and $M$. tuberculosis $\mathrm{H}_{37} \mathrm{Rv}$ (control) were carried out with $1 \mu \mathrm{L}$ of each mycobacterial DNA $(10 \mathrm{ng})$ in $24 \mu \mathrm{L}$ of a reaction mixture containing $2.5 \mu \mathrm{M}$ of each primer ERIC1R (ATGTAAGCTCCTGGGGATTCAC) and ERIC2F AAGTAAGTGACTGGGGTGAGCG) and PCR Master Mix (Promega Corporation, Madison, Wisconsin, USA) according to manufacturer's instructions. The amplification was carried out in a thermocycler (Applied Biosystems ${ }^{\circledR}$ Veriti ${ }^{\circledR}$ Thermal Cycler) programmed to $94{ }^{\circ} \mathrm{C}$ for 2 min and 35 cycles of $94{ }^{\circ} \mathrm{C}$ for $45 \mathrm{sec}, 52^{\circ} \mathrm{C}$ for $1 \mathrm{~min}, 70^{\circ} \mathrm{C}$ for $10 \mathrm{~min}$ and finally $70{ }^{\circ} \mathrm{C}$ for $20 \mathrm{~min}$.

The PCR amplicons were subjected to electrophoresis in $2.0 \% \mathrm{~W} / \mathrm{v}$ agarose gel (Invitrogen Life Technologies, São Paulo, Brazil). The 100-bp DNA Ladder (Invitrogen Life Technologies, São Paulo, Brazil) was used as molecular marker. Gel was stained with ethidium bromide $0.5 \mu \mathrm{g} / \mu \mathrm{L}$, visualized under an ultraviolet light Macrovue (Pharmacia Bioscience, Upsala, Sweden) and photodocumented digitally by Power Shot S215 (Cannon, NY, USA).

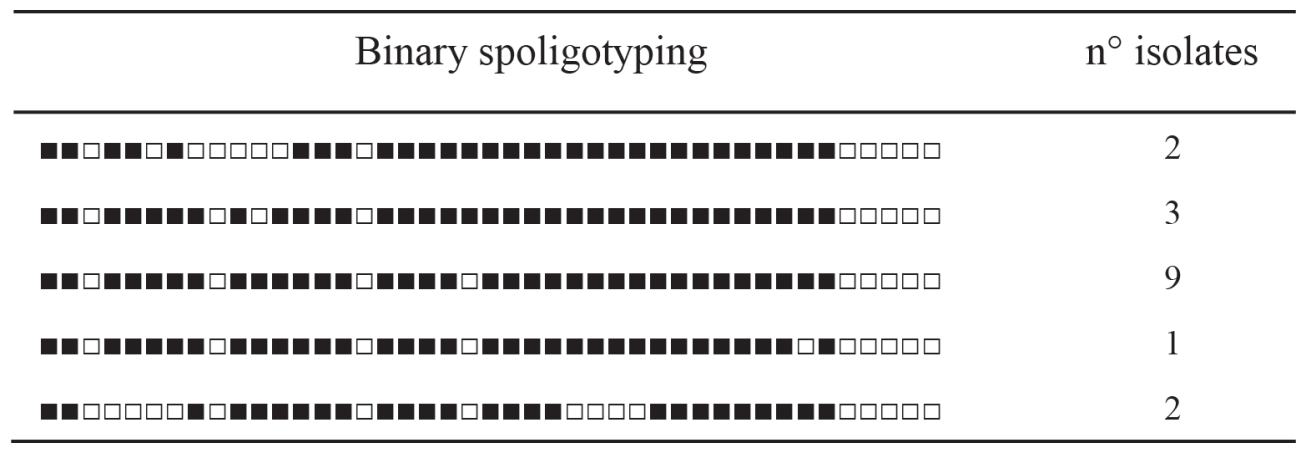

FIGURE 1 - Binary spoligotyping data of 17 Mycobacterium bovis isolates from lymph node samples collected from bovines with macroscopic tuberculous-like lesions or positive skin test during postmortem inspection in an abattoir in the northwestern region of Paraná State, Brazil. 


\section{Analysis}

BioNumerics software (version 4.45; Applied Maths, Sint-Martens-Latem, Belgium) was used for analysis of multi-band patterns in ERIC-PCR and compared to the Spoligotyping and 12 loci-MIRU, previously performed (Machado et al., 2014; Noguti et $a l ., 2010)$. Dendrograms were constructed and the genetic distance was built employing the UPGMA algorithm (Unweighted Pair Group Method with Arithmetic Mean) based on 1\% optimization and position tolerance for the three methods (Sneath, Sokal, 1973). Only isolates with $100 \%$ similarity were considered closely related.

The evaluation of the discriminative power of each typing method, separately as well as, in combination was undertaken by using the Hunter-Gaston index (HGI) (Hunter, Gaston, 1988), which is based on the probability that two unrelated strains sampled from the population test will be placed into different typing groups.

\section{RESULTS}

A comparison of the discriminatory power of ERICPCR to Spoligotyping and MIRU can be seen at Table I. ERIC-PCR, of 73 isolates from northwestern Paraná previously typed by Spoligotyping, rendered 59 patterns, in which 48 were unique and 11 clusters ( 24 isolates) with 2-4 isolates each one. The application of Spoligotyping plus ERIC-PCR in 73 isolates could not differentiate only 2 clustered isolates The analysis of 63 isolates genotyped by 12 loci-MIRU plus ERIC-PCR showed $100 \%$ of differentiation (Figure 2). The three methods rendered 100 $\%$ of differentiation of the isolates.

ERIC-PCR, carried out in 83 isolates from southwestern Parana, rendered 41 ERIC patterns. From these, 25 were unique and 16 clusters (58 isolates) with 2-9 isolates each one.

The analyses of 83 isolates typed by 12 loci-MIRU plus Spoligotyping could not differentiate only 4 clustered isolates in 2 clusters. Spoligotyping plus ERIC-PCR showed total differentiation of isolates in 6 of 11 clusters by Spoligotyping. The other isolates in the five clusters had partially differentiation of isolates. The application of 12 loci-MIRU plus ERIC-PCR could not differentiate only 2 clustered isolates (Figure 3). The three methods rendered $100 \%$ of differentiation of the isolates. ERIC-PCR, of 17 M. bovis isolates, rendered $100 \%$ of differentiation.

\section{DISCUSSION}

The present study was designed to compare

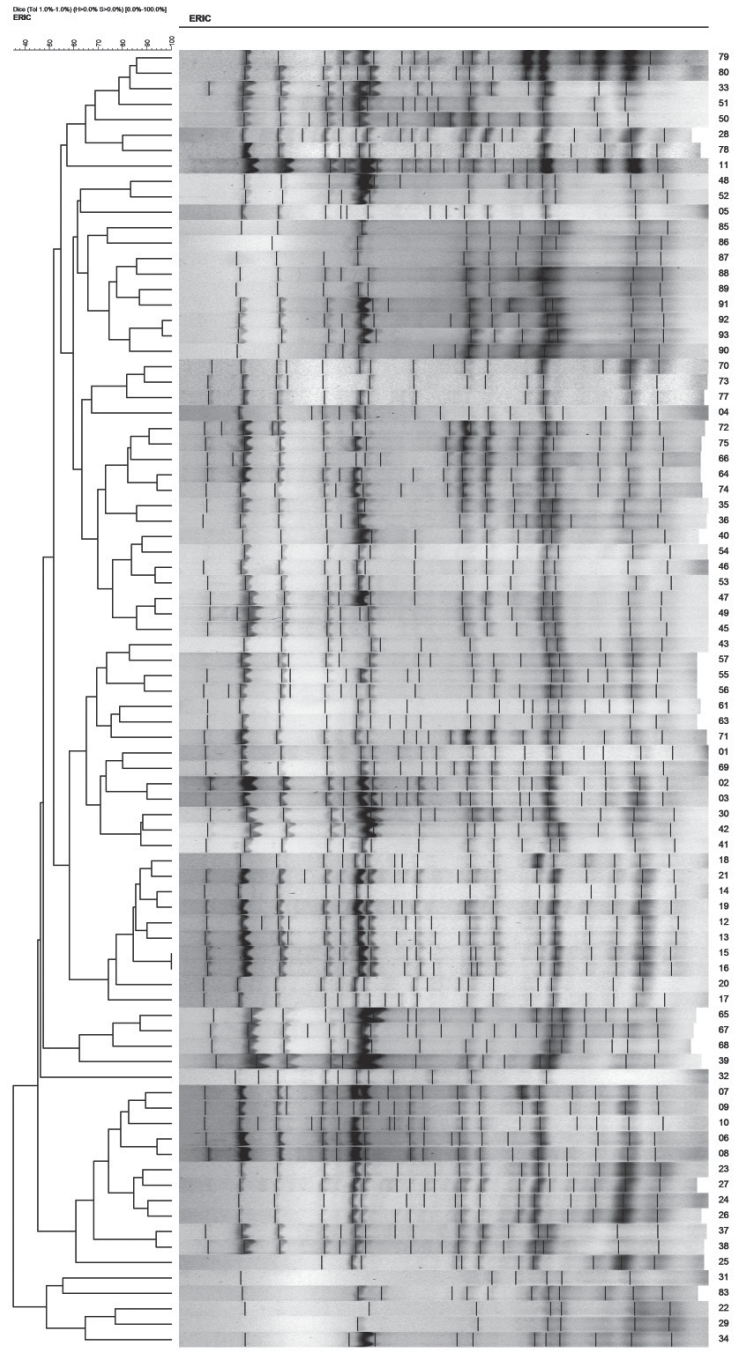

FIGURE 2 - Combined numerical analysis of Mycobacterium tuberculosis clinical isolates from northwestern, Paraná, Brazil, using Enterobacterial Repetitive Intergenic Consensus sequence (ERIC-PCR). The dendrogram was obtained using the BioNumerics software (version 4.45; Applied Maths, SintMartens-Latem, Belgium).

a very simple and fast genotyping method, ERICPCR, with Spoligotyping and MIRU to differentiate epidemiologically related from unrelated M. tuberculosis isolates. We feel this need since ERIC-PCR was a genotyping method that had been used previously, 10 years ago, in Mycobacterium tuberculosis and mycobacteria other than tuberculosis. From that, no study was carried out with ERIC-PCR in M. tuberculosis once other very used and established genotyping methods for epidemiological studies in tuberculosis showed to be useful, such as Spoligotyping and MIRU. At the time, ERIC-PCR has been used for genotyping different Gram negative bacillus. As ERIC-PCR is fast and an inexpensive method, it could be used as a screening tool in low-income laboratories to 


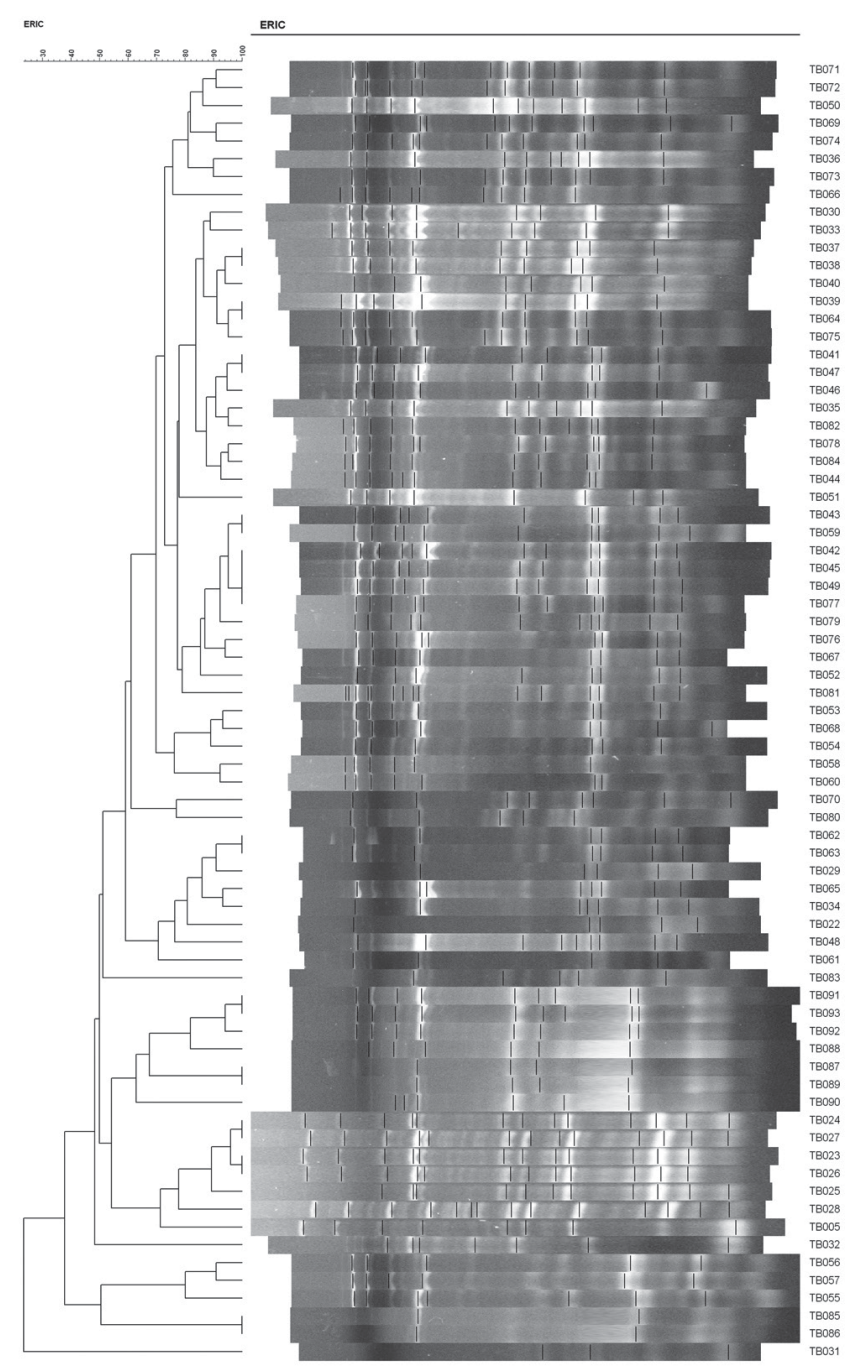

FIGURE 3 - Combined numerical analysis of Mycobacterium tuberculosis clinical isolates from southwestern, Paraná, Brazil, using Enterobacterial Repetitive Intergenic Consensus sequence (ERIC-PCR). The dendrogram was obtained using the BioNumerics software (version 4.45; Applied Maths, SintMartens-Latem, Belgium).

differentiate isolates from a suspected outbreak in little time.

The $M$. tuberculosis isolates studied were from two different settings, northwestern and southwestern Parana, Brazil. In the northwestern, patients belonged to eight cities (Maringá and seven small cities surrounding) which is considered a low-endemic area of TB in Parana, with a TB incidence rate of 22.62/100.000 (Noguti et al., 2010). In the southwestern, which borders the Paraguay and Argentina countries, patients were from two major cities, Foz do Iguaçu and Cascavel, and other eight small surrounding cities at the Brazilian border, with a TB incidence rate of 41.8/100.000 and 25.5/100.000, respectively (Machado et al., 2014).
In our study, comparing the performance of the three methods used separately and in combination as genotyping tools in M. tuberculosis, the classical 12 loci-MIRU showed better performance for genotypic differentiation of isolates from southwestern setting (HGI 0.997), which had higher incidence of TB compared with the northwestern setting. Nonetheless, ERIC-PCR was the best method for differentiating isolates from the northwestern setting (HGI 0.994).

The ERIC-PCR Electrophoretic analysis of the M. tuberculosis generated multiple bands with variable intensities. We observed banding patterns that had 1 to 12 bands with molecular sizes ranging from 100 to 1600 bp (Figure 2). Our analyses were carried out only with fragments below $1600 \mathrm{bp}$, which showed better resolution. The distribution of the fragments occurred evenly, but the majority was concentrated at $600 \mathrm{bp}$ region. As there are no established criteria for interpreting ERIC-PCR, what could cause difficulty to interpret the band patterns, we considered isolates to be different, according to Pellegrino et al. (2002), based on a single band difference and additional support analysis by the other two used genotyping methods (Spoligotyping and MIRU).

ERIC-PCR had the ability to differentiate isolates that were clustered by 12 loci-MIRU and Spoligotyping separately or combined. The combination of ERICPCR with 12 loci-MIRU showed to be equal to 12 lociMIRU plus Spoligotyping (HGI 0.999) to differentiate isolates from southwestern setting. In isolates from the northwestern, ERIC-PCR with 12 loci-MIRU (HGI 1.0) as well as ERIC-PCR with Spoligotyping (HGI 0.999) showed good discrimination of isolates, which suggests the high value in using two or more methods for genotypic studies in TB. Our results came in agreement with Sechi et al. (1998) and indicate that the ERIC sequence could be used as an independent marker, once the relationship among the profiles obtained by ERIC-PCR and those obtained by the available genotyping method for $M$. tuberculosis were not related. In the study conducted by Noguti et al. (2010), there were two isolates.

Cangelosi et al. (2004), Ashworth et al. (2008) and Jang et al. (2011) evaluated the utility of a commercially available rep-PCR System, initially developed for genotyping Streptococcus pneumoniae, Staphylococcus aureus, Enterobacteriaceae and others microorganism, to genotype M. tuberculosis and Mycobacterium avium Complex and found the system to be highly discriminative.

In contrast to the 12 loci-MIRU and Spoligotyping methods tested in our study, ERIC-PCR requires only one PCR per sample. 12 loci-MIRU requires 12 separately PCR and Spoligotyping one PCR followed by 
hybridization reaction. Thus, in terms of time spent, ERICPCR is very fast compared to the others. The same analysis can be made about the cost of the three methods, in which ERIC-PCR proved to be the less expensive.

Also, in our study, ERIC-PCR showed to be useful in differentiating M. bovis, species from the M. tuberculosis complex, which showed $100 \%$ of differentiation of isolates clustered by Spoligotyping. Although, our results came in agreement with Sechi et al. (1999), a study with a larger number of $M$. bovis isolates would be of great interest to understand the real utility of ERIC-PCR as genotypic tool for this species.

One limitation of our study is that none of the genotyped isolates were members of the Beijing family, which is not common in our region. In this sense, additional studies would contribute to know if ERIC-PCR has the ability in discriminating members of this family and also among them, which is very common in Asian countries with high incidence of TB.

As for any laboratory methodology, the reproducibility of the results depends on the standardization of each step during its execution. In the relevant literature, with other bacteria, no standardization on the DNA concentration was used to perform ERIC-PCR in most studies. In our experience with ERIC-PCR, standardization of the mycobacterial DNA concentration should be carried out to have more constant intensities of the DNA bands in the gel analyzes. Also, reagents and equipment should also be of the same manufacturer. In our study, we did not standardize the concentration of DNA from isolates to perform ERIC-PCR inasmuch as they were from previous studies (Machado et al., 2014; Noguti et al., 2010) and were stored at $-20^{\circ} \mathrm{C}$. As the ERIC-PCR were performed in duplicate by independent assays for the same isolates in different days, to assure its reproducibility, we could observe that no problem to read the gels occurred with the DNA bands with the most intensity. However, in a few cases, the bands with low intensity were difficult to be observed in the gel.

As the ERIC sequence, is not a proprietary technology and the primers and PCR conditions are commercially available, additional studies with isolates from different countries would help to elucidate the applicability of ERIC-PCR in outbreaks, which require fast resolution, and could after be completed with MIRU and/or Spoligotyping methods.

The molecular typing method has been used for a variety of epidemiologic investigations, such as those to confirm outbreaks in institutions and to identify them in situations that appear to be sporadic cases of TB, risk factors for recent infections or rapid progression diseases, tracking the geographical distribution of M. tuberculosis clusters of importance to public health and crosslaboratory contamination assessment. An ideal genotyping method should generate conclusive information in a fashion time for allowing direct interventions. However, to date, there is no method that fully meets qualities as to be simple, reproducible and affordable to be applied in many laboratories. In this context, ERIC-PCR could, maybe, fill these qualities. In conclusion, we observed that ERICPCR would be an important tool in the epidemiology of TB as a screening in outbreaks, which demand rapid intervention. However if any doubt persists, as it may occur with the application of only one genotypic method, other disposable genotyping methods for this purpose should be applied and carefully interpreted always with additional epidemiological information.

\section{ACKNOWLEDGEMENTS}

This work was supported by Coordenação de Aperfeiçoamento de Pessoal de Nível Superior (CAPES) and Conselho Nacional de Desenvolvimento Científico e Tecnológico (CNPq).

\section{CONFLICT OF INTEREST}

No conflict of interest declared.

\section{REFERENCES}

Ashworth M, Horan KL, Freeman R, Oren E, Narita M, Cangelosi GA. Use of PCR-based Mycobacterium tuberculosis genotyping to prioritize tuberculosis outbreak control activities. J Clin Microbiol. 2008;46(3):856-862.

Cangelosi GA, Freeman RJ, Lewis KN, Livingston-Rosanoff D, Shah KS, Milan SJ, et al. Evaluation of a high-throughput repetitive-sequence-based PCR system for DNA fingerprinting of Mycobacterium tuberculosis and Mycobacterium avium complex strains. J Clin Microbiol. 2004;42(6):2685-2693.

Englund S. IS900/ERIC-PCR as a tool to distinguish Mycobacterium avium subsp. paratuberculosis from closely related mycobacteria. Vet Mirobiol. 2003;96(3):277-287.

González-Y-Merchand J, Estrada-García I, Colston MJ, Cox RA. A novel method for the isolation of mycobacterial DNA. FEMS Microbiol Lett. 1996;135(1):71-77.

Hunter PR, Gaston MA. Numerical index of the discriminatory ability of typing systems: an application of Simpson's index of diversity. J Clin Microbiol. 1988;26(11):2465-2466. 
Jang MH, Choi GE; Bo-Moon S, Seon HL, Sung-Ryul K, Chulhun L, et al. Comparison of an Automated Repetitive Sequence-based PCR Microbial Typing System with IS6110Restriction Fragment Length Polymorphism for Epidemiologic Investigation of Clinical Mycobacterium tuberculosis Isolates in Korea. Korean J Lab Med. 2011;31(4):282-284.

Kent PT, Kubica G. Public Health Mycobacteriology: a guide for a level III laboratory. Atlanta, GA: US Department of Health and Human Services, Centers for Disease Control and Prevention; 1985.

Machado LNC, Marcondes NR, Leite CQF, Santos ACB, Pavan FR, Baldin VP, et al. First Baseline of Circulating Genotypic Lineages of Mycobacterium tuberculosis in Patients from the Brazilian Borders with Argentina and Paraguay. PLoS One 2014;9(9):e107106.

Mears J, Abubakar I, Cohen T, McHugh TD, Sonnenberg P. Effect of study design and setting on tuberculosis clustering estimates using Mycobacterial Interspersed Repetitive UnitsVariable Number Tandem Repeats (MIRU-VNTR): a systematic review. BMJ Open. 2015;5(1):e005636.

Noguti ENA, Leite CQF, Malaspina AC, Santos ACB, Hirata RDC, Hirata MH, et al. Genotyping of Mycobacterium tuberculosis isolates from alow-endemic setting in northwestern state of Paraná in Southern Brazil. Mem Inst Osw Cruz. 2010;105(6):779-785.

Pandolfi JR, Malaspina AC, Santos ACB, Suffys PN, Oellemann MAC, Valentini SR, et al. Tuberculose e o estudo molecular da sua epidemiologia. Rev Ciênc Farm Básica Apl. 2007;28(3):251257.

Pellegrino FL, Teixeira LM, Carvalho MDGS, Aranha Nouér S, Pinto De Oliveira M, Mello Sampaio JL, et al. Occurrence of a multidrug-resistant Pseudomonas aeruginosa clone in different hospitals in Rio de Janeiro, Brazil. J Clin Microbiol. 2002;40(7):2420-2424.

Sampaio JLM, Chimara E, Ferrazoli L, Silva Telles MA, Del Guercio VMF, Jericó ZVN, et al. Application of four molecular typing methods for analysis of Mycobacterium fortuitum group strains causing post-mammaplasty infections. Clin Microbiol Infect. 2006a;12(2):142-149.
Sampaio JLM, Viana-Niero C, Freitas D, Hofling-Lima AL, Leão SC. Enterobacterial repetitive intergenic consensus PCR is an useful tool for typing Mycobacterium chelonae and Mycobacterium abscessus isolates. Diag Microbiol Infet Diseases. 2006b;55(2):107-118.

Sechi LA, Leori G, Lollai SA, Duprè I, Molicotti P, Fadda $\mathrm{G}$, et al. Different strategies for molecular differentiation of Mycobacterium bovis strains isolated in Sardinia, Italy. Appl Environ Microbiol. 1999;65(4):1781-1785.

Sechi LA, Zanetti S, Dupré I, Delogu G, Fadda G. Enterobacterial Repetitive Intergenic Consensus Sequences as Molecular Targets for Typing of Mycobacterium tuberculosis Strains. J Clin Microbiol. 1998;36(1):128-132.

Sneath PHA, Sokal RR. Numerical taxonomy: the principles and practice of numerical classification. San Francisco: WH Freeman \& Co; 1973.

Supply P, Lesjean S, Savine E, Kremer K, Soolingen D, Locht C. Aumtomated high-throughtput genotyping for study of global epidemiology of Mycobacterium tuberculosis based on Mycobacterial interspersed repetitive units. J Clin Microbiol. 2001;39(10):3563-3571

Supply P, Mazars E, Lesjean S, Vincent V, Gicquel B, Locht C. Variable human minisatellite-like regions in the Mycobacterium tuberculosis genome. Molec Microbiol. 2000;36(3):762-771.

Versalovic J, Koeuth T, Lupski JR. Distribution of repetitive DNA Sequences in eubacteria and application to fingerprint of bacterial genomes. Nucleic Acids Res 1991;19:6823-6831.

World Health Organization. WHO. Global tuberculosis control: WHO 2016 Report, Geneva: World Health Organization; 2017.

Received for publication on $11^{\text {th }}$ September 2017 Accepted for publication on $17^{\text {th }}$ January 2018 